\title{
Mobile Drone Localization in Indoor Environment Based on Passive RFID
}

https://doi.org/10.3991/ijim.v14i05.13309

\author{
Mohamed Hadi Habaebi ( $\left.{ }^{\varpi}\right)$, Rashid Khamis Omar, Md Rafiqul Islam \\ International Islamic University Malaysia, Kuala Lumpur, Malaysia \\ habaebi@iium.edu.my
}

\begin{abstract}
Radio Frequency Identification (RFID) is an information exchange technology based on RF communication. It provides solution to track and localize mobile objects in the indoor environment. Localization of mobile objects in an indoor environment garnered a significant attention due to the variety of applications needing higher degree of localization accuracy. RSSbased localization techniques are the major tools for tracking applications due to their simplicity. In this paper, a trilateration method for indoor localization is proposed. This method provides a solution for the drone tracking problem by collecting the RSS values between RFID tagged drone and reader, and estimate its location. The localization method is implemented in MATLAB by multiple readers; 4 RFID readers and 8 RFID readers. The performance of the localization method is also compared with other RFID localization previously reported in the literature. The simulation results in the case of 8 RFID readers demonstrate more accurate results than 4 RFID readers by minimizing the localization error from 0.84606 to $0.40079 \mathrm{~m}$. The results also indicate an improved localization performance of tracking a tagged drone in indoor environment by $42.8 \%$ when 8 RFID readers are placed in the localization area.
\end{abstract}

Keywords - RFID, Indoor Localization, Trilateration, Received Signal Strength.

\section{Introduction}

In the last decade, the wireless sensing technology has led the development to implement the RFID in a wide range of application such as supply chain management [1], industrial automation [2], mining securities [3] and localization and tracking system [4]. Many systems of tracking and localization of a mobile object have been widely studied in the literatures. However, most of the techniques such as GPS are not suitable for indoor applications due to the weak signals. Therefore, the issue of a drone localization system that uses RFID tags in indoor environment has been one among the interesting topics for research.

Yang, et al. [5], proposed an object tracking algorithm in an indoor environment, consisting of locating RFID reader with passive RFID tags used as landmarks in the environment. Thus, they used a hybrid method that combines PF and WCL to reduce 
the localization error at much lower cost, however, if the object speed is kept low. The simulation is performed in the scenario in which the tag spacing is set to 0.27 meters, the reader space is 0.3 meters and the average localization error is 0.062 meters. In [6] examined the issue of indoor navigation system that allows the user to independently move to the grid of UHF RFID tags for localization. The experiment is conducted in a laboratory environment. UHF RFID tags are placed on the ceiling at a height of 3 meters and constitute a square mesh area of 1.2 meters space, then localization error achieved is less than $0.5 \mathrm{~m}$.

In this paper, we study the problem of localization which can be used to localize the tagged drone using 4 and 8 RFID readers in indoor environment. The RFID readers are attached to the ceiling of the coverage area and their corresponding coordinates which are measured in advance. The RFID reader captured the RSS values from the tagged drone which are used to calculate the distance between the reader and the RFID tagged drone by using trilateration approach. The remainder of the paper is organized as follows. Next section provides localization system model. Section 3 shows the results where actual and estimated coordinates are presented. Finally we conclude our work in section 4.

\section{Localization System Model}

The RFID based localization system consists of three components: RFID reader, passive RFID tags and environment infrastructures [7]. Figure 1 shows the localization system that is connected by RFID readers and antennas. Assume there are $n$ number of passive RFID reference tags which are used in the coverage area and RFID readers, the localization system estimates the position of the drone per interval of time $t$. It is assumed that there is no dead zone of the reference tags, the reader antenna within the coverage area collect the set of known reference tag IDs and the set of the RSS values measured for each reference tag [8].

RSS values of the reference tags which are attached to the ceiling are interfered with the drone flight because it is assumed that the drone flies at a low height from the ground. The height of the drone can be measured by the ultra sound sensor that is available beneath the drone. The localization method continues to make the reader, for certain duration of time, for collect and identify the reference tags IDs and the RSS values. Whenever the drone moves near to the reference tags or away from a reference tag, the trajectory of the drone are produced. In this way, the method can track the position of the drone by selecting the reference tags which are mostly captured by the drone. With the use of the coordinates of the RFID readers which are stored in advance, the method can analyse the variation of the RSS values for each reference to track and estimate the location of the drone. Therefore, this paper proposes the trilateration method based on RSS technique that uses estimation distance to localize the target drone. 


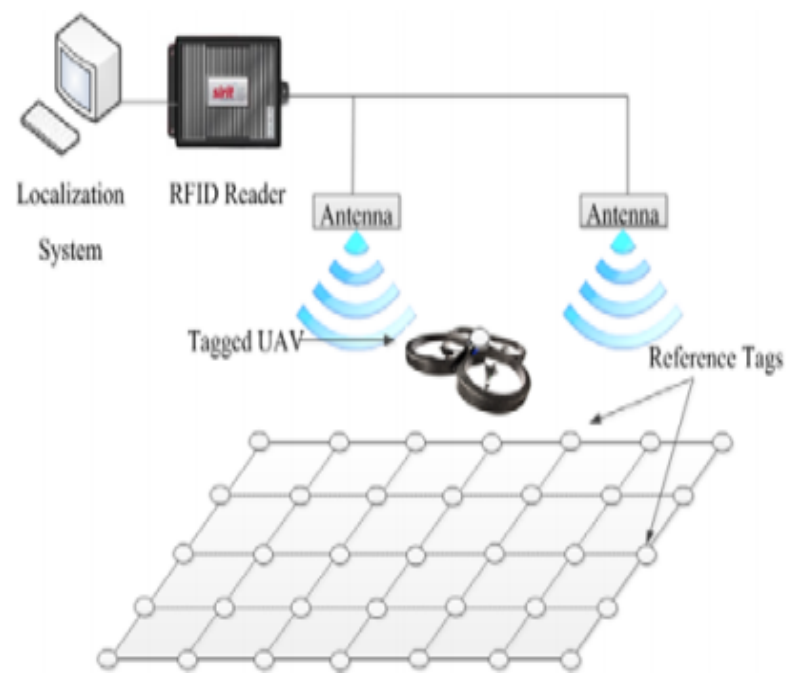

Fig. 1. Localization System Model

\subsection{RSS based localization}

The RSS based localization basically applies an appropriate path loss model to localize the target RFID tag in indoor environment, there are different RFID systems which are used for the localization of the RFID tags. Some of the RFID systems use number of measurements of reads per second as a distance measure, considering that the RFID tag is often read when it is close to the reader. The target RFID tag location can be estimated based on the measurements of multiple readers at different locations by combining the distance measurements of three readers in a fixed location with the help of trilateration method. The target RFID tag location estimate can be influenced by the errors in the antenna location, changing of the reader output power and the measurements of the distance. [9].Equation (1) shows a direct relation between distance and the received signal strength (RSS) measured in decibel (dB). The RSS is an indicator, consistent with non-physical characteristics of received signal difference, which is the actual power level.

$$
D=10^{\frac{A-R S S}{10 n}}
$$

Where $D$ is the distance between reader and tag, $n$ is the propagation path-loss and $A$ is the received signal strength at one-meter distance.

\subsection{Trilateration method}

The localization algorithm proposed in this paper is the RSS based trilateration method. This method presumed that all the readers in the localization area are at fixed locations. Each of these readers transmit the RF signals to the target tagged drone, when 
the tagged drone detects the RF signal it becomes active and the antenna of the tagged drone reflects back the RSS to the RFID readers. Each of these readers converted the RSS to distance by using Equation (1). The coordinates' locations of each of these RFID readers are known in advance. The location of target tagged drone is calculated based on the measurement from readers in the localization area. At least three measurements of readers distance from the tagged drone are needed in order to estimate the target tagged drone. Figure 2 shows the requirement concept of trilateration method, the intersection of three spheres determine the location of unknown tagged drone in localization area where the radii of the spheres are the distances from each reader to the target tagged drone and the respective location of each reader is the centre of each sphere.

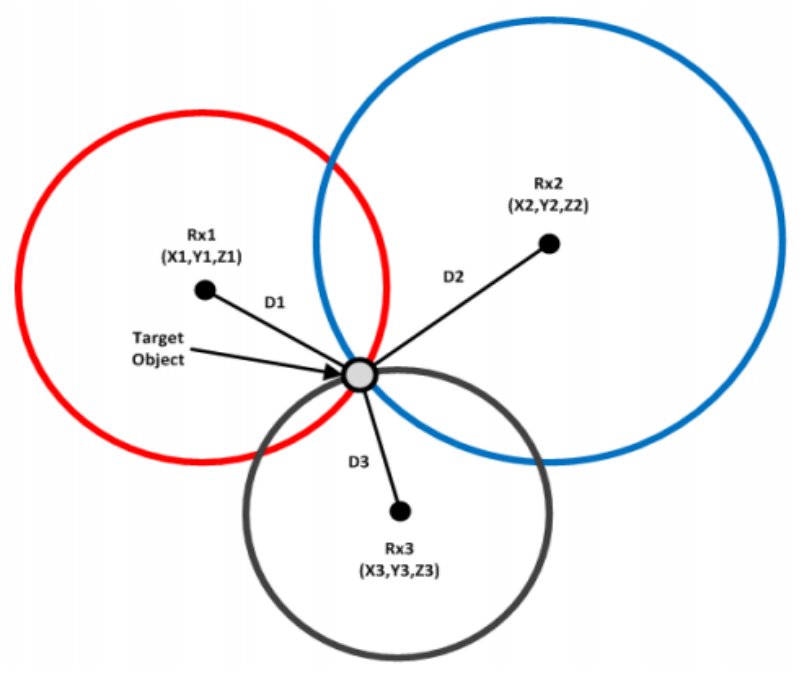

Fig. 2. Trilateration method

The relationship between the fixed location of the three readers and the target tagged drone are obtained after approximating the distance between them, are shown in Equations (2), (3) and (4).

$$
\begin{aligned}
& D_{1}^{2}=\left(x-x_{1}\right)^{2}+\left(y-y_{1}\right)^{2}+\left(z-z_{1}\right)^{2} \\
& D_{2}^{2}=\left(x-x_{2}\right)^{2}+\left(y-y_{2}\right)^{2}+\left(z-z_{2}\right)^{2} \\
& D_{3}^{2}=\left(x-x_{3}\right)^{2}+\left(y-y_{3}\right)^{2}+\left(z-z_{3}\right)^{2}
\end{aligned}
$$

where $(x, y, z)$ is the tagged drone coordinates to be estimated, $\left(x_{i}, y_{i}, z_{i}\right)$ is the location of three readers and $D_{i}$ are the predicted distance from the tagged drone to the three readers. Consider all the readers located on the same plane, by letting $z=0$, the three 
readers vertices $(1,2$ and 3$)$ have distances $\left(D_{1}, D_{2}, D_{3}\right)$ to the target object, as shown in Figure 2.

Equations (2), (3) and (4) can further be expanded to the following equations:

$$
\begin{aligned}
& D_{1}^{2}=x^{2}+x_{1}^{2}-2 x x_{1}+y^{2}+y_{1}^{2}-2 y y_{1} \\
& D_{2}^{2}=x^{2}+x_{2}^{2}-2 x x_{2}+y^{2}+y_{2}^{2}-2 y y_{2} \\
& D_{3}{ }^{2}=x^{2}+x_{3}{ }^{2}-2 x x_{3}+y^{2}+y_{3}{ }^{2}-2 y y_{3}
\end{aligned}
$$

Equations (5), (6) and (7) are independent non-linear equations which cannot be solved mathematically. However, [7] proposed method which can be used to obtain the intersection point of the sphere, equation (7) was subtracted from equation (6) to obtain the following equation:

$$
\begin{aligned}
& D_{2}^{2}-D_{3}^{2}=2 x\left(x_{3}-x_{2}\right)+x_{2}^{2}-x_{3}^{2}+2 y\left(y_{3}-y_{2}\right)+y_{2}^{2}-y_{3}^{2} \\
& D_{2}^{2}-D_{1}^{2}=2 x\left(x_{1}-x_{2}\right)+x_{2}^{2}-x_{1}^{2}+2 y\left(y_{1}-y_{2}\right)+y_{2}^{2}-y_{1}^{2}
\end{aligned}
$$

Further subtracting equation (5) from equation (6), yields the following equation: Rearranging the equation (8) to get a new equation and a new variable as follows,

$$
x\left(x_{3}-x_{2}\right)+y\left(y_{3}-y_{2}\right)=\frac{\left(D_{2}^{2}-D_{3}^{2}\right)-\left(x_{2}^{2}-x_{3}^{2}\right)-\left(y_{2}^{2}-y_{3}^{2}\right)}{2}=v_{1}
$$

Rearranging the equation (9) to get a new equation and a new variable as follows:

$$
x\left(x_{1}-x_{2}\right)+\left(y_{1}-y_{2}\right)=\frac{\left(D_{2}^{2}-D_{1}^{2}\right)-\left(x_{2}^{2}-x_{1}^{2}\right)-\left(y_{2}^{2}-y_{1}^{2}\right)}{2}=v_{2}
$$

Solve the equations (10) and (11) to obtain the intersection point $\mathrm{x}$ and $\mathrm{y}$ as follows:

$$
\begin{gathered}
x=\frac{v_{1}-y\left(y_{3}-y_{2}\right)}{\left(x_{3}-x_{2}\right)} \\
y=\frac{v_{2}\left(x_{3}-x_{2}\right)-v_{1}\left(x_{1}-x_{2}\right)}{\left(y_{1}-y_{2}\right)\left(x_{3}-x_{2}\right)-\left(y_{3}-y_{2}\right)\left(x_{1}-x_{2}\right)}
\end{gathered}
$$

The values of $\mathrm{x}$ and $\mathrm{y}$ provides the actual location in the localization area for the tagged drone. 


\subsection{Procedure for estimated drone location}

The structure of a localization area for estimating the drone location is shown in Figure 3. Considering that there are four RFID readers and $n$ reference tags in the localization area, each reader is located at fixed position on the same plane. The locations of the readers form a rectangle, and at least three readers locations are needed in order to apply the trilateration method and one reader must be at the origin.

The tag $R_{l}$ has $R S S$ values $S_{11}, S_{21}, S_{31}, S_{41}$ from the four readers and its corresponding coordinates are $\left(x_{1}, y_{l}\right)$. However, for the localization system that consist of $m$ readers and $n$ reference tags, the values of RSS are recorded as $R S S=\left[S_{11}, S_{12}, S_{13}, \ldots, S_{I n}\right]$, where $S_{i}$ is the RSS value of readings from $m$ readers of the $i_{t h}$ reference tag, and their corresponding distances are denoted as $D=\left[D_{1}, D_{2}, D_{3}, \ldots . ., \mathrm{Dn}\right]$, where Di is the distance of the $\mathrm{m}$ readers to the ith reference tag. Figure 3 shows the flow chart of proposed method of drone localization.

\subsection{Algorithm}

Step1: Start procedure at Readers

Step2: Repeat, Move to a new distance for each $\mathrm{D}=1$ to 10

Transmission of information including its coordinate at the time until finish

Step3: End procedure

Step4: procedure at Tagged drone

Repeat

Receive information from readers

Calculate D according to equation (1)

Until finish

Set the readers information MAN at time ti

For all information MAN at the same time

If $\mid$ MAN $\mid>=3$

Estimate the location according to the equations (12) and (13)

Else

Discard MAN

End

Calculate the localization error of all estimated location according to (14)

Step5: End procedure. 


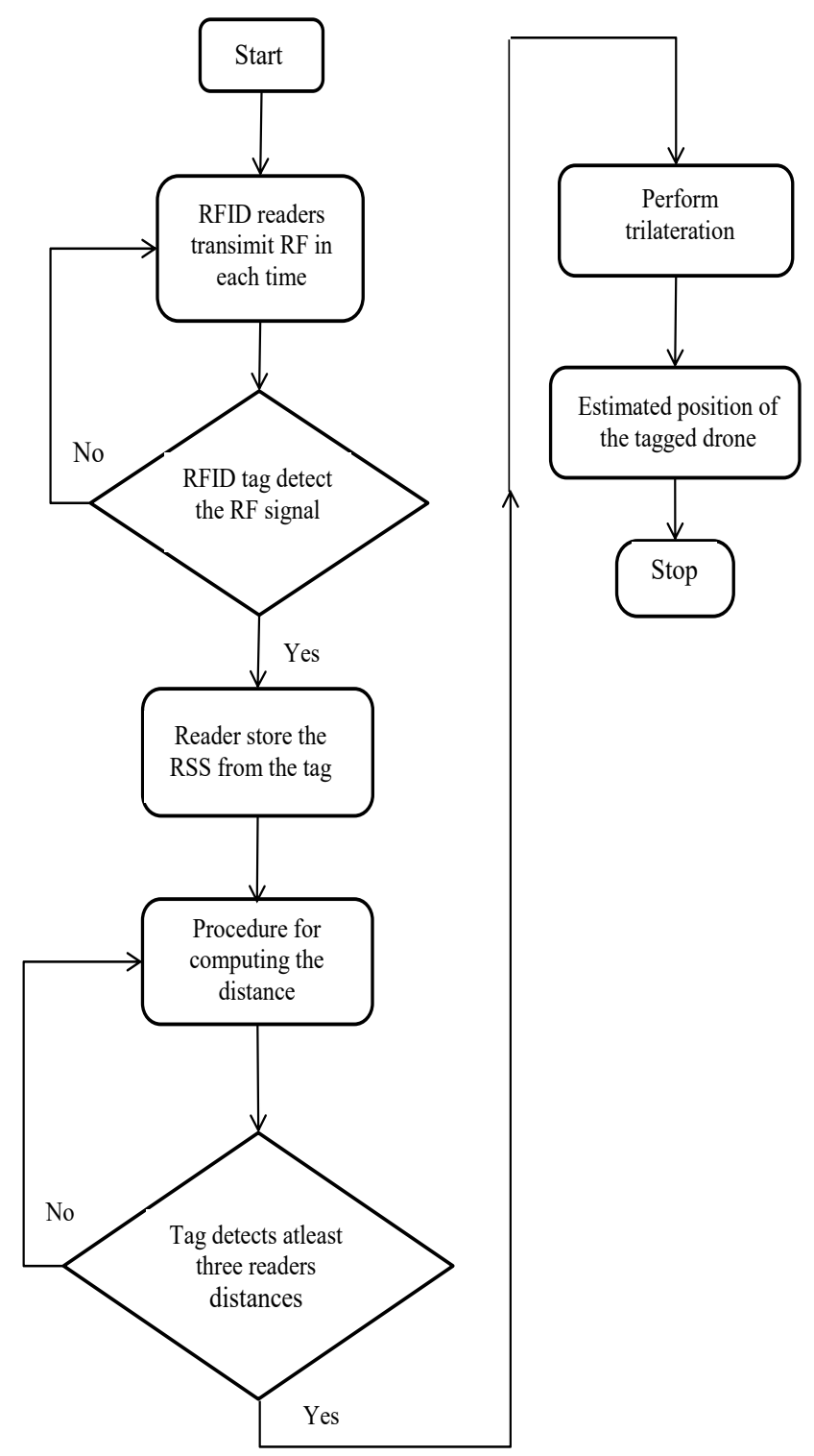

Fig. 3. Flow chart of proposed method of drone localization 


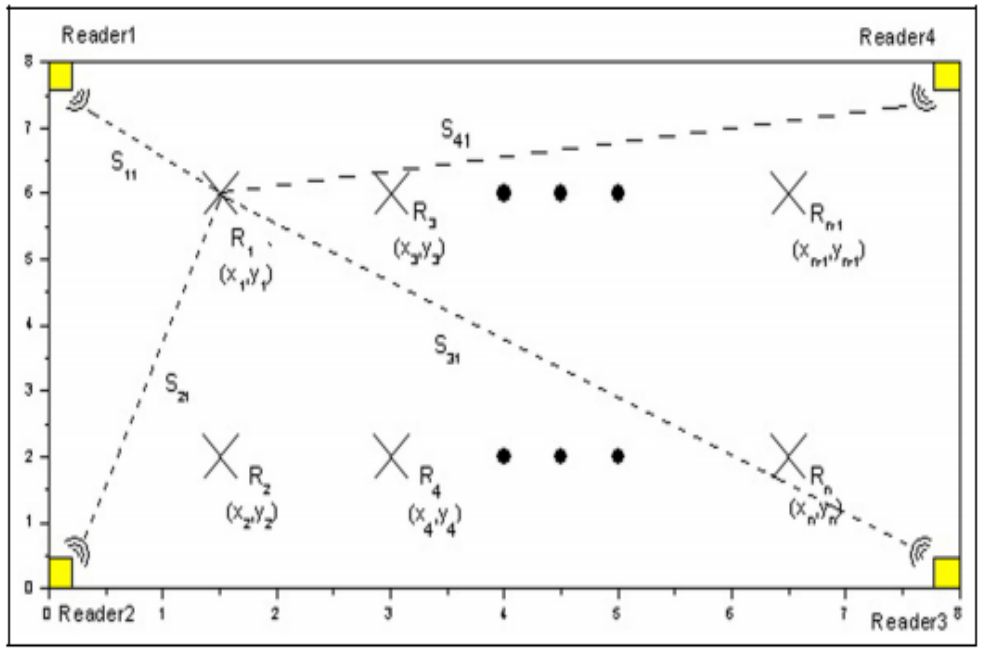

Fig. 4. Procedure for estimated drone location

\subsection{Performance metric}

Performance of the localization and tracking method is assessed on the basis of the localization errors. The choice of this metric depends on the idea that it measures localization accuracy. Since the method aims to localize the drone in the indoor environment, and improve the efficiency of the current method with respect to localization accuracy, the localization error is determined to ensure that the localization method has achieved the desired goal, by finding an optimal solution of $(\mathrm{x}, \mathrm{y})$ that reduces the sum of squared differences between the distances and their corresponding values. Equation (4.1) shows the final localization error (LE), between the estimated location $(\mathrm{x}, \mathrm{y}$,$) and the actual location \left(\mathrm{x}^{\wedge}, \mathrm{y}^{\wedge}\right)$ of the tagged drone.

$$
L E=\sqrt{\left(x-x^{\wedge}\right)^{2}+\left(y-y^{\wedge}\right)^{2}}
$$

\section{Performance Evaluation of Drone Location Estimation}

The simulations results are performed by using two different numbers of multiple RFID readers which are 4 and 8 in the localization area of $10 \times 10$ and localization error (LE) value for each number of multiple readers are calculated. To compare the performance of these multiple RFID readers, Figure 4 and 5 shows the localization accuracy resulted from 4 multiple RFID readers and 8 multiple RFID readers, respectively. The black circle represents the actual location of RFID readers, while the red one indicates the estimated location of the drone and the plus sign represents the actual location of the drone. 


\subsection{Four RFID readers location estimation performance}

From Figure 4, the localization area was covered by $2 \times 2$ RFID readers, and the transmission range between RFID readers were $10 \mathrm{~m}$. The RFID readers antenna were placed facing down to the ground. The RFID readers activated the antenna which were more close from the distance of the tagged drone in the simulation. It can be observed that, the difference between the actual and estimated location is high. This is due to weak signal levels of the RSS measurement which were affected by strong multipath propagation and larger distance between the readers. The localization error achieved for an estimated drone location is $0.84606 \mathrm{~m}$.

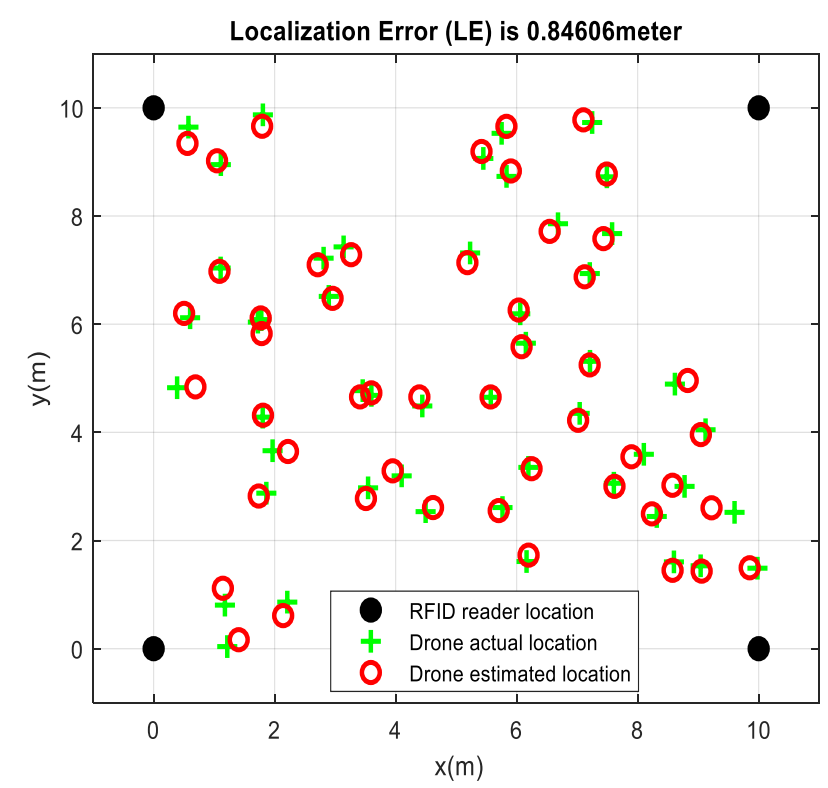

Fig. 5. The estimated location and the actual location of the tagged drone with 4 RFID readers

\subsection{Eight RFID readers location estimation performance}

In order to improve the localization accuracy, number of eight RFID readers was doubled in the localization area as shown in Figure 5. The localization area was covered by $3 \times 3$ RFID readers and the transmission range was decreased from 10 to $5 \mathrm{~m}$ in all directions. The graph shows that the localization method has achieved the highest accuracy for locating the tagged drone this is due to the improvement of the signal power of the RSS where this improvement has been caused by a decrease in the distance between readers and tags. The localization error achieved is about 0.40079 meter for a $5 \mathrm{~m}$ transmission range of the readers, a reduction by half from the case $\mathrm{o} 4$ RFID readers only. 


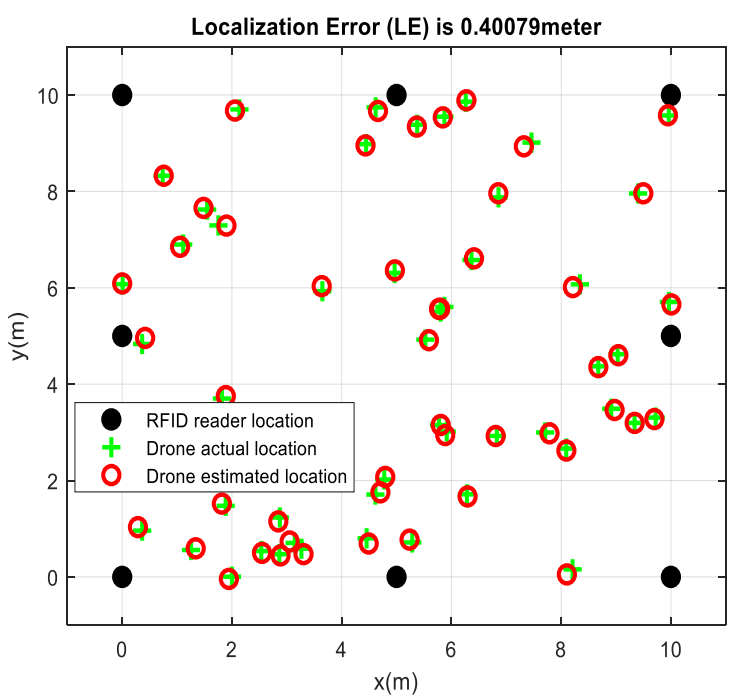

Fig. 6. The estimated location and the actual location of the tagged drone with 8 RFID readers

The localization method has shown that, increasing of the number of RFID readers has resulted decreases the localization error. The increased number of RFID readers in the system has therefore improved the localization accuracy of the drone. In this case, the localization accuracy was improved to about $52.62 \%$.

To further verify the proposed localization method, its compared with the previous results from other related works, Yang, et al., (2015) observing the object tracking and localization in indoor environment, consisting of a locating RFID readers with passive RFID tags used as landmark in the environment. The localization error of about 0.602 meter achieved. Similar localization range, as for Hongshan\& Yu (2018), the experiment is conducted in laboratory environment. The tags are placed on the ceiling at height $3 \mathrm{~m}$ and $1.2 \mathrm{~m}$ for reader space, then localization error achieved is $0.5 \mathrm{~m}$. Another RFID system technique for target localization is a method of detection probability (Geng, et al., 2014). It is reported that the localization error achieved is about $1.0 \mathrm{~m}$ with Particle Filter (PF) method. Therefore, compared with these reported results, the localization method proposed in this paper is efficient, promises good location estimation accuracy and can be easily implemented in indoor environment.

\section{Conclusion}

In this paper, we have studied the problem of localizing the tagged drone with the RSS technique based on trilateration approach using 4 RFID readers and 8 RFID readers in indoor environment. The simulation results in the case of 8 RFID readers show more accurate results than 4 RFID readers by minimizing the localization error from 0.84606 to 0.40079 meter. The performance of the localization method is also compared with other RFID localization previous related works. The results have shown 
that the performance in localization of tagged drone in indoor environment has been improved by $52.62 \%$ when 8 multiple RFID readers are applied in the localization area.

There are still many research problems in the issue of the RSS-based trilateration localization, such as detecting sudden changes in the direction of the mobile target, varying speed of the mobile target and further working on the concepts of RFID tags and readers on the information exchange can improve the accuracy of the mobile target localization problem in indoor environment.

\section{Acknowledgement}

This work was conducted at the IOT and Wireless Communication Protocols Laboratory, International Islamic University Malaysia (IIUM) and is partially funded by IIUM Publication Grant P-RIGS18-003-0003.

\section{References}

[1] ALSALIH, W., ALMAAITAH, A., \& ALKHATER, W. (2014). RFID Localization Using Angle of Arrival Cluster Forming. In International Journal of Disributed Sensor Networks Vol 2014, 8 pages. https://doi.org/10.1155/2014/269596

[2] YU, J., CHEN, L., \& WANG, K. (2017). On Missing Tag Detection in Multiple-Group Multiple-Region RFID Systems. In IEEE Transactions on Mobile Computing. https://doi.org/10.1109/TMC.2016.2592902

[3] RUAN, W., YAO, L., \& SHENG, Z. (2017). Tagtrack: Device Free Localization and Tracking Using Passive RFID Tags. School of computer science, The university of Adelaide, Australia

[4] M.H. HABAEBI, R.O. KHAMIS, W., YAO, L., \& MD. R. ISLAM (2014). RSS Based Localization Techniques for Zigbee Wireless Sensor Network. International Conference on Computer and Communication Enginerring. https://doi.org/10.1109/ICCCE.2014.32

[5] YANG, L., CAO, J., \& ZHU, W. (2015). Accurate and efficient object tracking based on passive RFID. In IEEE transactions on mobile computing, Vol 14, November 2015. https://doi.org/10.1109/TMC.2014.2381232

[6] KANG, J., \& KIM, H. (2015). Analysis of Localization for Drone-Fleet. In IEEE conference paper, Dept of information and communication engineering. https://doi.org/10.1109/ICTC.2015.7354604

[7] OGUEJIOFOR O. S, ANIEDU A.N, EJIOFOR H.C (2013). Trilateration Based Localization Algorithm for Wireless Sensor Network. International Journal of Science and Modern Engineering. Volume-1, Issue-10, Sept 2013.

[8] KONG, H., \& YU, BIN (2018). A Moving Object Indoor Tracking Model Based on Semiactive RFID. In mathematical problems in engineering Vol.2018 7pages. Zhengzhou, China. https://doi.org/10.1155/2018/4812057

[9] EL-ABSI, M., ABBAS, A., \& ABUELHAIJA, A. (2018). High-Accuracy Indoor Localization Based on Chipless RFID Systems at Thz Band. In IEEE access. September 2018. https://doi.org/10.1109/ACCESS.2018.2871960

[10] GENG, L., BUGALLO, F., \& ATHALYSE, A. (2014). Indoor Tracking with RFID Systems. In IEEE Journal of selected topics in signal processing, Vol 8, feb 2014. https://doi.org/10.1109/JSTSP.2013.2286972 
[11] HUITING, J. (2017) Indoor Localization of UHF RFID tags. Computer architecture design and test for embedded system.

\section{$7 \quad$ Authors}

Mohamed Hadi Habaebi is a professor with the department of Electrical and Computer Engineering, International Islamic University Malaysia. His research interests are in IoT, wireless communications, small antenna design, wireless channel propagation, FSO propagation and Networking.

Email: habaebi@iium.edu.my

Rashid Khamis Omar is pursuing his Master of Science in Communication Engineering at the International Islamic University Malaysia. His research interests are towards the fields of Telecommunications, Internet of Things, Wireless Sensor Networks, Drone technologies and Electronics. E-mail: rashidrok@hotmail.com

Md Rafiqul Islam is a professor with the department of Electrical and Computer Engineering, International Islamic University Malaysia. His research interests are in wireless channel modeling, radio link design, RF propagation measurement and modeling in tropical and desert, RF design, smart antennas and array antennas design, FSO propagation and modeling etc. Email: rafiq@iium.edu.my

Article submitted 2020-01-07. Resubmitted 2020-02-21. Final acceptance 2020-02-22. Final version published as submitted by the authors. 